\title{
On the Existence and Uniqueness of $R_{v}$-Generalized Solution for Dirichlet Problem with Singularity on All Boundary
}

\author{
V. Rukavishnikov and E. Rukavishnikova \\ Computing Center of Far-Eastern Branch, Russian Academy of Sciences, Kim-Yu-Chen Street 65, Khabarovsk 680000, Russia \\ Correspondence should be addressed to V. Rukavishnikov; vark0102@mail.ru
}

Received 20 January 2014; Revised 19 May 2014; Accepted 16 June 2014; Published 3 July 2014

Academic Editor: Donal O’Regan

Copyright (c) 2014 V. Rukavishnikov and E. Rukavishnikova. This is an open access article distributed under the Creative Commons Attribution License, which permits unrestricted use, distribution, and reproduction in any medium, provided the original work is properly cited.

\begin{abstract}
The existence and uniqueness of the $R_{v}$-generalized solution for the first boundary value problem and a second order elliptic equation with coordinated and uncoordinated degeneracy of input data and with strong singularity solution on all boundary of a two-dimensional domain are established.
\end{abstract}

\section{Introduction}

The singularity of solution for boundary value problems to two-dimensional closed domain can be due to the degeneration of the input data (coefficients and right-hand sides of equations and boundary conditions), availability of the reentrant corners, and change of the kind of the boundary conditions or by the internal properties of the solution. A boundary value problem is said to possess strong singularity if its solution $u(x)$ does not belong to Sobolev space $W_{2}^{1}\left(H^{1}\right)$ or, in other words, the Dirichlet integral of the solution $u(x)$ diverges. In the case if the solution belongs to the space $W_{2}^{1}$ $\left(H^{1}\right)$ but does not belong to the space $W_{2}^{2}\left(H^{2}\right)$, a boundary value problem is called the problem with a weak singularity.

Boundary value problems with strong singularity are found in the physics of plasma and gas discharge, electrodynamics, nuclear physics, nonlinear optics, and other branches of physics. In particular cases, numerical methods for problems of electrodynamics and quantum mechanics with strong singularity were constructed, based on separation of singular and regular components, mesh refinement near singular points, multiplicative extraction of singularities, and so forth, (see, e.g., [1-6]).

In [7], it was suggested to define the solution of boundary value problem for second-order elliptic equation with singularity on a finite set of points belonging to boundary of a two-dimensional domain as an $R_{v}$-generalized solution in the weighted Sobolev space. Such a new concept of solution led to the distinction of two classes of boundary value problems: problems with coordinated and uncoordinated degeneracy of input data; it also made it possible to study the existence and uniqueness of solutions as well as its coercivity and differential properties in the weighted Sobolev spaces (see $[8,9])$.

For boundary value problems for elliptic equations, Maxwell equations and Lamé system, we constructed the numerical methods with rate of convergence independent of the singularity based on the concept of an $R_{\gamma}$-generalized solution (see, e.g., [10-12]).

In this paper, we consider the first boundary value problem for a second-order elliptic equation with strong singularity solution on all boundary of a two-dimensional domain. We distinguish two classes of the boundary value problems: problems with coordinated and uncoordinated degeneracy of input data. For this problem we define the solution as an $R_{v}$-generalized one in a weighted Sobolev space $H_{2, \nu+\beta / 2}^{1}(\Omega)$ and in a weighted set $W_{2, \nu+\beta / 2}^{1}(\Omega, \delta)$, respectively. We prove its existence and uniqueness in the corresponding weighted space and weighted set. It was established that, for all values of parameter $v$ for which the $R_{v}$-generalized solution exists, it is unique for all of these parameters. 


\section{Notation and Auxiliary Statements}

We denote the two-dimensional Euclidean space by $\mathbb{R}^{2}$ with $x=\left(x_{1}, x_{2}\right)$ and $d x=d x_{1} d x_{2}$. Let $\Omega \subset \mathbb{R}^{2}$ be a bounded domain with sufficiently smooth boundary $\partial \Omega$, and let $\bar{\Omega}$ be the closure of $\Omega$; that is, $\bar{\Omega}=\Omega \cup \partial \Omega$. We denote by $\Omega^{\prime}$ the adjoining streak of the boundary $\partial \Omega$ of width $\delta>0$ and $\Omega^{\prime} \subset$ $\Omega$.

We introduce a weight function $\rho(x)$ that coincides in $\Omega^{\prime}$ with the distance from point $x$ to the boundary $\partial \Omega$ and is equal to $\delta$ for $x \in \bar{\Omega} \backslash \Omega^{\prime}$.

Let $H_{2, \alpha}^{k}(\Omega)$ and $W_{2, \alpha}^{k}(\Omega)$ be the weighted spaces with norms:

$$
\begin{gathered}
\|u\|_{H_{2, \alpha}^{k}(\Omega)}=\left(\sum_{|\lambda| \leq k} \int_{\Omega} \rho^{2(\alpha+|\lambda|-k)}\left|D^{\lambda} u\right|^{2} d x\right)^{1 / 2}, \\
\|u\|_{W_{2, \alpha}^{k}(\Omega)}=\left(\sum_{|\lambda| \leq k} \int_{\Omega} \rho^{2 \alpha}\left|D^{\lambda} u\right|^{2} d x\right)^{1 / 2},
\end{gathered}
$$

where $D^{\lambda}=\partial^{|\lambda|} / \partial x_{1}^{\lambda_{1}} \partial x_{2}^{\lambda_{2}}, \lambda=\left(\lambda_{1}, \lambda_{2}\right)$, and $|\lambda|=\lambda_{1}+$ $\lambda_{2} ; \lambda_{1}, \lambda_{2}$ are integer nonnegative numbers, $\alpha$ is some real nonnegative number, and $k$ is an integer nonnegative number. For $k=0$ we use the notation $H_{2, \alpha}^{0}(\Omega)=W_{2, \alpha}^{0}(\Omega)=L_{2, \alpha}(\Omega)$.

By $W_{2, \alpha}^{1}(\Omega, \delta)$ for $\alpha>0$, we denote a set of functions satisfying the following conditions:

(a) $\left|D^{k} u(x)\right| \leq c_{1}(\delta / \rho(x))^{\alpha+k}$ for $x \in \Omega^{\prime}$, where $k=0,1$, $c_{1}$ is positive constant independent of $k$,

(b) $\|u\|_{L_{2, \alpha}\left(\Omega \backslash \Omega^{\prime}\right)} \geq c_{2}>0$,

and with the norm (2).

The spaces $\stackrel{\circ}{H}_{2, \alpha}^{k}(\Omega) \subset H_{2, \alpha}^{k}(\Omega)$ and $\stackrel{\circ}{W}_{2, \alpha}^{k}(\Omega) \subset W_{2, \alpha}^{k}(\Omega)$ and the set $\dot{W}_{2, \alpha}^{1}(\Omega, \delta) \subset W_{2, \alpha}^{1}(\Omega, \delta)$ are defined as the closures of the set of infinitely differentiable and finite in $\Omega$ functions in norms (1) and (2), respectively.

Let $H_{\infty,-\alpha}^{k}\left(\Omega, c_{3}\right)(k \geq 0, \alpha \in R)$ be the set of functions with the norm satisfying the inequality

$$
\|u\|_{H_{\infty,-\alpha}^{k}\left(\Omega, c_{3}\right)}=\max _{|\lambda| \leq k} \operatorname{ess} \sup _{x \in \Omega}\left|\rho^{-\alpha+|\lambda|} D^{\lambda} u\right| \leq c_{3}
$$

with a positive constant $c_{3}$ independent of $u$. For $k=0$, we have $H_{\infty,-\alpha}^{0}\left(\Omega, c_{4}\right)=L_{\infty,-\alpha}\left(\Omega, c_{4}\right)$.

Lemma 1. For each function $u$ in the set $W_{2, \alpha}^{1}(\Omega, \delta)$ and for any $\alpha^{*}>\alpha$, the estimate

$$
\|u\|_{L_{2, \alpha^{*}-1}\left(\Omega^{\prime}, \delta\right)} \leq \mathcal{C}_{5}\|u\|_{L_{2, \alpha^{*}}(\Omega, \delta)}
$$

holds, where $c_{5}=c_{6}\left(\delta^{\alpha} / \sqrt{\alpha^{*}-\alpha}\right), c_{6}=$ const $>0$.
Proof. Taking into account condition (a), one can show that, for $\alpha^{*}>\alpha$, we have

$$
\begin{aligned}
\|u\|_{L_{2, \alpha^{*}-1}\left(\Omega^{\prime}, \delta\right)}^{2} & =\int_{\Omega^{\prime}} \rho^{2\left(\alpha^{*}-1\right)} u^{2} d x \\
& \leq c_{1}^{2} \delta^{2 \alpha} \int_{\Omega^{\prime}} \rho^{2\left(\alpha^{*}-1\right)} \rho^{-2 \alpha} d x \\
& \leq \frac{c_{1}^{2} \delta^{2 \alpha} c_{7} \delta^{2\left(\alpha^{*}-\alpha\right)}}{2\left(\alpha^{*}-\alpha\right)},
\end{aligned}
$$

where $c_{7}$ is a constant dependent of mes $\Omega^{\prime}$. Considering condition (b), we write the inequality for the function $u$ as follows:

$$
\begin{aligned}
\|u\|_{L_{2, \alpha^{*}}(\Omega)}^{2} & \geq\|u\|_{L_{2, \alpha^{*}}\left(\Omega \backslash \Omega^{\prime}\right)}^{2} \\
& =\delta^{2\left(\alpha^{*}-\alpha\right)}\|u\|_{L_{2, \alpha}\left(\Omega \backslash \Omega^{\prime}\right)}^{2} \geq c_{2}^{2} \delta^{2\left(\alpha^{*}-\alpha\right)} .
\end{aligned}
$$

From inequalities (5) and (6) we get the estimate (4) with $c_{6}=$ $\left(c_{1} / c_{2}\right) \sqrt{c_{7} / 2}$.

\section{The Boundary Value Problem with Coordinated Degeneration of the Input Data on All Boundary of the Domain}

In the domain $\Omega$, we consider the differential equation

$$
\begin{gathered}
-\sum_{k, l=1}^{2} a_{k l}(x) \frac{\partial^{2} u}{\partial x_{k} \partial x_{l}}+\sum_{k=1}^{2} a_{k}(x) \frac{\partial u}{\partial x_{k}} \\
+a(x) u=f(x), \quad x \in \Omega,
\end{gathered}
$$

with the boundary condition

$$
u=0, \quad x \in \partial \Omega .
$$

Definition 2. The boundary value problem (7) and (8) is called the Dirichlet problem with coordinated degeneration of the input data on all boundary of the domain or Problem $\mathrm{A}$, if $a_{k l}(x)=a_{l k}(x)(k, l=1,2)$ and, for some real number $\beta$,

$$
\begin{gathered}
a_{k l} \in H_{\infty,-\beta}^{1}\left(\Omega, c_{8}\right), \quad a_{k} \in L_{\infty,-(\beta-1)}\left(\Omega, c_{9}\right), \quad(k, l=1,2), \\
a \in L_{\infty,-(\beta-2)}\left(\Omega, c_{10}\right),
\end{gathered}
$$

$$
\sum_{k, l=1}^{2} a_{k l}(x) \xi_{k} \xi_{l} \geq c_{11} \rho^{\beta}(x) \sum_{k=1}^{2} \xi_{k}^{2},
$$

$$
a(x)>c_{12} \rho^{\beta-2}(x) \text { almost everywhere on } \Omega
$$

and right-hand side of (7) satisfies

$$
f \in L_{2, \mu}(\Omega),
$$

where $c_{i}(i=8, \ldots, 12)$ are positive constants independent of $x ; \xi_{1}$ and $\xi_{2}$ are any real parameters; $\mu$ is some nonnegative real number. 
Denote by

$$
\begin{gathered}
a(u, v)=\int_{\Omega}\left[\sum_{k, l=1}^{2} a_{k l} \rho^{2 v} \frac{\partial u}{\partial x_{k}} \frac{\partial v}{\partial x_{l}}+a_{k l} \frac{\partial \rho^{2 v}}{\partial x_{k}} \frac{\partial u}{\partial x_{l}} v\right. \\
+\frac{\partial a_{k l}}{\partial x_{k}} \rho^{2 v} \frac{\partial u}{\partial x_{l}} v+a_{k} \rho^{2 v} \frac{\partial u}{\partial x_{k}} v \\
\left.+a \rho^{2 v} u v\right] d x \\
f(v)=\int_{\Omega} \rho^{2 v} f v d x
\end{gathered}
$$

the bilinear and linear forms, respectively.

Definition 3. A function $u_{v}$ from the space $\stackrel{\circ}{H}_{2, v+\beta / 2}^{1}(\Omega)$ is called an $R_{v}$-generalized solution of the Dirichlet problem with coordinated degeneration of the input data on all boundary of the domain or Problem A, if, for any $v$ in $\stackrel{\circ}{H}_{2, v+\beta / 2}^{1}(\Omega)$, the identity

$$
a\left(u_{v}, v\right)=f(v)
$$

holds, where $v$ is arbitrary but fixed and satisfies the inequality

$$
v \geq \mu+\frac{\beta}{2}-1
$$

For Problem A, we prove the main result.

Theorem 4. Let conditions (9)-(12) and (15) hold and let

$$
2\left(c_{8}(2|\nu|+1)+\frac{1}{2} c_{9}\right)^{2}<c_{11} c_{12}
$$

be satisfied.

Then, the $R_{v}$-generalized solution $u_{v}$ of the Dirichlet problem with coordinated degeneration of the input data on all boundary of the domain exists and is unique in the space $\stackrel{\circ}{H}_{2, v+\beta / 2}^{1}(\Omega)$ and the following estimate is valid:

$$
\left\|u_{\nu}\right\|_{H_{2, \nu+\beta / 2}^{1}(\Omega)} \leq c_{13}\|f\|_{L_{2, \mu}(\Omega)},
$$

where $c_{13}$ is a positive constant not depending on $u_{v}$ and $f$.

Proof. First, we show that the forms $a(u, v)$ and $f(v)$ are continuous on $\stackrel{\circ}{H}_{2, \nu+\beta / 2}^{1}(\Omega)$. In fact, by virtue of conditions (9), (12), and (15) and the Cauchy-Schwarz inequality, we have

$$
\begin{gathered}
|a(u, v)| \\
\leq \int_{\Omega} \mid \sum_{k, l=1}^{2} a_{k l} \rho^{2 v} \frac{\partial u}{\partial x_{k}} \frac{\partial v}{\partial x_{l}}+\frac{\partial a_{k l}}{\partial x_{k}} \rho^{2 v} \frac{\partial u}{\partial x_{l}} v \\
+a_{k} \rho^{2 v} \frac{\partial u}{\partial x_{k}} v+a \rho^{2 v} u v \mid d x
\end{gathered}
$$

$$
\begin{aligned}
& +\int_{\Omega^{\prime}}\left|a_{k l} \frac{\partial \rho^{2 v}}{\partial x_{k}} \frac{\partial u}{\partial x_{l}} v\right| d x \\
& \leq 2 c_{8}\left(\int_{\Omega} \rho^{2 v+\beta} \sum_{k=1}^{2}\left(\frac{\partial u}{\partial x_{k}}\right)^{2} d x\right)^{1 / 2} \\
& \times\left(\int_{\Omega} \rho^{2 v+\beta} \sum_{k=1}^{2}\left(\frac{\partial v}{\partial x_{k}}\right)^{2} d x\right)^{1 / 2} \\
& +2 \sqrt{2} c_{8}\left(\int_{\Omega} \rho^{2 v+\beta} \sum_{l=1}^{2}\left(\frac{\partial u}{\partial x_{l}}\right)^{2} d x\right)^{1 / 2} \\
& \times\left(\int_{\Omega} \rho^{2 v+\beta-2} v^{2} d x\right)^{1 / 2} \\
& +\sqrt{2} c_{9}\left(\int_{\Omega} \rho^{2 v+\beta} \sum_{k=1}^{2}\left(\frac{\partial u}{\partial x_{k}}\right)^{2} d x\right)^{1 / 2} \\
& \times\left(\int_{\Omega} \rho^{2 v+\beta-2} v^{2} d x\right)^{1 / 2}+c_{10}\left(\int_{\Omega} \rho^{2 v+\beta-2} u^{2} d x\right)^{1 / 2} \\
& \times\left(\int_{\Omega} \rho^{2 v+\beta-2} v^{2} d x\right)^{1 / 2} \\
& +4 \sqrt{2}|\nu| c_{8}\left(\int_{\Omega^{\prime}} \rho^{2 v+\beta} \sum_{l=1}^{2}\left(\frac{\partial u}{\partial x_{l}}\right)^{2} d x\right)^{1 / 2} \\
& \times\left(\int_{\Omega^{\prime}} \rho^{2 v+\beta-2} v^{2} d x\right)^{1 / 2} \\
& \leq 2 c_{8}|u|_{H_{2, v+\beta / 2}^{1}(\Omega)}|v|_{H_{2, v+\beta / 2}^{1}(\Omega)} \\
& +\left(2 \sqrt{2} c_{8}+\sqrt{2} c_{9}\right)|u|_{H_{2, \gamma+\beta / 2}^{1}(\Omega)}\|v\|_{L_{2, v+\beta / 2-1}(\Omega)} \\
& +4 \sqrt{2}|\nu| c_{8}|u|_{H_{2, \gamma+\beta / 2}^{1}\left(\Omega^{\prime}\right)}\|v\|_{L_{2, v+\beta / 2-1}\left(\Omega^{\prime}\right)} \\
& +c_{10}\|u\|_{L_{2, v+\beta / 2-1}(\Omega)}\|v\|_{L_{2, v+\beta / 2-1}(\Omega)},
\end{aligned}
$$

or

$$
|a(u, v)|
$$

$$
\leq c_{14}\|u\|_{H_{2, v+\beta / 2}^{1}(\Omega)}\|v\|_{H_{2, v+\beta / 2}^{1}(\Omega)}, \quad \forall u, v \in \stackrel{\circ}{H}_{2, v+\beta / 2}^{1}(\Omega)
$$

$|f(v)|$

$$
\begin{aligned}
& \leq\left(\max _{x \in \Omega} \rho^{2 v-2 \mu-\beta+2}\right)^{1 / 2}\left(\int_{\Omega} \rho^{2 \mu} f^{2} d x\right)^{1 / 2} \\
& \times\left(\int_{\Omega} \rho^{2 v+\beta-2} v^{2} d x\right)^{1 / 2} \leq c_{15}\|f\|_{L_{2, \mu}(\Omega)}\|v\|_{H_{2, \nu+\beta / 2}^{1}(\Omega)}, \\
& \forall v \in \stackrel{\circ}{H}_{2, \nu+\beta / 2}^{1}(\Omega), \forall f \in L_{2, \mu}(\Omega) .
\end{aligned}
$$


Let us now prove the $\stackrel{\circ}{H}_{2, v+\beta / 2}^{1}(\Omega)$ ellipticity of the bilinear form $a(u, v)$; that is,

$$
\exists c_{16}>0, \quad \forall u \in \stackrel{\circ}{H}_{2, v+\beta / 2}^{1}(\Omega), \quad a(u, u) \geq c_{16}\|u\|_{H_{2, v+\beta / 2}^{1}}^{2}(\Omega) .
$$

We substitute $v$ by $u$ in (13), and by means of the CauchySchwarz inequality, $\varepsilon$-inequality, and conditions (9), we estimate the absolute values of the second, third, and fourth terms of the form $a(u, u)$ :

$$
\begin{aligned}
& \left|\sum_{k, l=1}^{2} \int_{\Omega} a_{k l} \frac{\partial \rho^{2 v}}{\partial x_{k}} \frac{\partial u}{\partial x_{l}} u d x\right| \\
& \leq\left|\sum_{k, l=1}^{2} \int_{\Omega} a_{k l} 2 v \rho^{2 v-1} \frac{\partial u}{\partial x_{l}} u d x\right| \\
& \leq 2 \sqrt{2} c_{8}|\nu|\left(\varepsilon_{1} \sum_{l=1}^{2} \int_{\Omega^{\prime}} \rho^{2 v+\beta}\left(\frac{\partial u}{\partial x_{l}}\right)^{2} d x\right. \\
& \left.+\frac{1}{\varepsilon_{1}} \int_{\Omega^{\prime}} \rho^{2 v+\beta-2} u^{2} d x\right), \\
& \left|\sum_{k, l=1}^{2} \int_{\Omega} \frac{\partial a_{k l}}{\partial x_{k}} \rho^{2 v} \frac{\partial u}{\partial x_{l}} u d x\right| \\
& \leq \sqrt{2} c_{8}\left(\varepsilon_{2} \sum_{l=1}^{2} \int_{\Omega} \rho^{2 v+\beta}\left(\frac{\partial u}{\partial x_{l}}\right)^{2} d x\right. \\
& \left.+\frac{1}{\varepsilon_{2}} \int_{\Omega} \rho^{2 v+\beta-2} u^{2} d x\right), \\
& \left|\sum_{k=1}^{2} \int_{\Omega} a_{k} \rho^{2 v} \frac{\partial u}{\partial x_{k}} u d x\right| \\
& \leq \sqrt{2} c_{9}\left(\frac{\varepsilon_{3}}{2} \sum_{k=1}^{2} \int_{\Omega} \rho^{2 v+\beta}\left(\frac{\partial u}{\partial x_{k}}\right)^{2} d x\right. \\
& \left.+\frac{1}{2 \varepsilon_{3}} \int_{\Omega} \rho^{2 \nu+\beta-2} u^{2} d x\right) .
\end{aligned}
$$

Here, $\varepsilon_{1}, \varepsilon_{2}$, and $\varepsilon_{3}$ are any positive numbers.

Using (10) and (11), we have

$$
\begin{gathered}
\sum_{k, l=1}^{2} \int_{\Omega} a_{k l} \rho^{2 v} \frac{\partial u}{\partial x_{k}} \frac{\partial u}{\partial x_{l}} d x \geq c_{11} \int_{\Omega} \rho^{2 v+\beta} \sum_{k=1}^{2}\left(\frac{\partial u}{\partial x_{k}}\right)^{2} d x \\
\int_{\Omega} a \rho^{2 v} u^{2} d x \geq c_{12} \int_{\Omega} \rho^{2 v+\beta-2} u^{2} d x
\end{gathered}
$$

Then, from (22)-(26), we obtain

$$
\begin{aligned}
& a(u, u) \\
& \geq\left(c_{11}-\sqrt{2}\left(c_{8} 2|\nu| \varepsilon_{1}+c_{8} \varepsilon_{2}+\frac{1}{2} c_{9} \varepsilon_{3}\right)\right) \\
& \quad \times \int_{\Omega} \rho^{2 v+\beta} \sum_{k=1}^{2}\left(\frac{\partial u}{\partial x_{k}}\right)^{2} d x \\
& \quad+\left(c_{12}-\sqrt{2}\left(c_{8} 2|\nu| \varepsilon_{1}^{-1}+c_{8} \varepsilon_{2}^{-1}+\frac{1}{2} c_{9} \varepsilon_{3}^{-1}\right)\right) \\
& \quad \times \int_{\Omega} \rho^{2 v+\beta-2} u^{2} d x .
\end{aligned}
$$

Note that if condition (16) is satisfied, then there exists a positive constant $\varepsilon$ such that

$$
\begin{gathered}
c_{11}-\sqrt{2}\left(c_{8}(2|\nu|+1)+\frac{1}{2} c_{9}\right) \varepsilon>0, \\
c_{12}-\sqrt{2}\left(c_{8}(2|\nu|+1)+\frac{1}{2} c_{9}\right) \varepsilon^{-1}>0 .
\end{gathered}
$$

Supposing that $\varepsilon_{1}=\varepsilon_{2}=\varepsilon_{3}=\varepsilon$ in (27), we get

$$
\begin{aligned}
& a(u, u) \\
& \geq c_{16}\left(\int_{\Omega} \rho^{2 v+\beta} \sum_{k=1}^{2}\left(\frac{\partial u}{\partial x_{k}}\right)^{2} d x\right. \\
& \left.\quad+\int_{\Omega} \rho^{2 v+\beta-2} u^{2} d x\right)=c_{16}\|u\|_{H_{2, \nu+\beta / 2}^{1}(\Omega)}
\end{aligned}
$$

with constant

$$
\begin{aligned}
c_{16}=\min \left(c_{11}-\sqrt{2}\left(c_{8}(2|\nu|+1)+\frac{1}{2} c_{9}\right) \varepsilon,\right. \\
\left.c_{12}-\sqrt{2}\left(c_{8}(2|\nu|+1)+\frac{1}{2} c_{9}\right) \varepsilon^{-1}\right) .
\end{aligned}
$$

According to (19), (21), and (20), the bilinear form $a(u, v)$ is continuous and $\stackrel{\circ}{H}_{2, v+\beta / 2}^{1}(\Omega)$-elliptical, and the linear form $f(v)$ is continuous on $\stackrel{\circ}{H}_{2, v+\beta / 2}^{1}(\Omega)$; then, the existence and uniqueness of an $R_{v}$-generalized solution of Problem A follow from the Lax-Milgram theorem (see [13]).

Taking into account that

$$
\begin{aligned}
c_{16}\left\|u_{\nu}\right\|_{H_{2, \nu+\beta / 2}^{1}(\Omega)}^{2} & \leq a\left(u_{v}, u_{v}\right) \\
& =f(v) \leq c_{15}\|f\|_{L_{2, \mu}(\Omega)}\|u\|_{H_{2, \nu+\beta / 2}^{1}(\Omega)},
\end{aligned}
$$

we get estimate (17).

Corollary 5. If there exists at least one $v$ for which there exists a unique $R_{v}$-generalized solution of the Problem $A$, then one can 
always define a half-open interval $\left[\nu_{1}, \nu_{2}\right)$ such that, for each $v \in\left[v_{1}, v_{2}\right)$, there exists a unique $R_{v}$-generalized solution. Here,

$$
\begin{gathered}
\nu_{1}=\max \left\{\mu+\frac{\beta}{2}-1 ;\left(1-\frac{\left(c_{11} c_{12}\right)^{1 / 2}-c_{9} / 2}{c_{8}}\right)+\varepsilon\right\}, \\
\nu_{2}=\frac{\left(c_{11} c_{12}\right)^{1 / 2}-c_{9} / 2}{c_{8}}-1,
\end{gathered}
$$

where $\varepsilon$ is a given sufficiently small positive number.

Corollary follows from the proof of Theorem 4.

Theorem 6. If the assumptions of Theorem 4 are valid, then, for all $v$ in the interval $\left[\nu_{1}, v_{2}\right)$, the $R_{v}$-generalized solution of the Problem $A$ is unique. in [14].

The proof of this statement is similar to that of Theorem 2

\section{The Boundary Value Problem with Uncoordinated Degeneration of the Input Data on All Boundary of the Domain}

We consider the boundary value problem

$$
\begin{gathered}
-\sum_{k=1}^{2} \frac{\partial}{\partial x_{k}}\left(a_{k k}(x) \frac{\partial u}{\partial x_{k}}\right)+a(x) u=f(x), \quad x \in \Omega, \\
u=0, \quad x \in \partial \Omega .
\end{gathered}
$$

Definition 7. The boundary value problem (33) and (34) is called the Dirichlet problem with uncoordinated degeneration of the input data on all boundary of the domain or Problem B, if, for some real number $\beta$,

$$
\begin{gathered}
a_{k k} \in H_{\infty,-\beta}^{1}\left(\Omega, c_{16}\right), \quad a \in L_{\infty,-\beta}\left(\Omega, c_{17}\right), \\
\sum_{k=1}^{2} a_{k k}(x) \xi_{k}^{2} \geq c_{18} \rho^{\beta}(x) \sum_{k=1}^{2} \xi_{k}^{2}, \\
a(x) \geq c_{19} \rho^{\beta}(x) \text { almost everywhere on } \Omega,
\end{gathered}
$$

and the right-hand side of the equation satisfies the condition

$$
f \in L_{2, \mu}(\Omega, \delta)
$$

for some nonnegative real number $\mu$. Here, $c_{i}, i=16, \ldots, 19$, are positive constants not depending on $x$; $\xi_{1}$ and $\xi_{2}$ are arbitrary real parameters.

Set

$$
\begin{gathered}
b(u, v)=\int_{\Omega}\left[\sum_{k=1}^{2} a_{k k} \rho^{2 v} \frac{\partial u}{\partial x_{k}} \frac{\partial v}{\partial x_{k}}\right. \\
\left.+a_{k k} \frac{\partial \rho^{2 v}}{\partial x_{k}} \frac{\partial u}{\partial x_{k}} v+a \rho^{2 v} u v\right] d x \\
l(v)=\int_{\Omega} \rho^{2 v} f v d x
\end{gathered}
$$

Definition 8. A function $u_{v}$ from the set $\stackrel{\circ}{W}_{2, v+\beta / 2}^{1}(\Omega, \delta)$ is called an $R_{v}$-generalized solution of the Problem $\mathrm{B}$ if the identity $b\left(u_{v}, v\right)=l(v)$ holds for all $v \in \stackrel{\circ}{W}_{2, v+\beta / 2}^{1}(\Omega, \delta)$ and for any given value of $v$ satisfying the inequality

$$
v \geq \mu+\frac{\beta}{2} .
$$

Theorem 9. Let conditions (35)-(40) hold and

$$
v+\frac{\beta}{2}>0
$$

Then, for any $v$ satisfying conditions (40) and (41), there always exists parameter $\delta$ such that $R_{v}$-generalized solution $u_{v}$ of the Dirichlet problem with uncoordinated degeneration of the input data on all boundary of the domain exists and is unique in the set $\stackrel{\circ}{W}_{2, v+\beta / 2}^{1}(\Omega, \delta)$. In this case, the following estimate is valid:

$$
\left\|u_{\nu}\right\|_{W_{2, \nu+\beta / 2}^{1}(\Omega, \delta)} \leq c_{20}\|f\|_{L_{2, \mu}(\Omega, \delta)}
$$

where $c_{20}$ is a positive constant independent of $f$ and $u_{v}$.

Proof. First of all, we note that the bilinear and linear forms are continuous on the set $\stackrel{\circ}{W}_{2, \nu+\beta / 2}^{1}(\Omega, \delta)$ and the inequalities

$$
\begin{gathered}
b(u, v) \leq c_{21}\|u\|_{W_{2, v+\beta / 2}^{1}(\Omega, \delta)}\|v\|_{W_{2, v+\beta / 2}^{1}(\Omega, \delta)}, \\
l(v) \leq c_{22}\|f\|_{L_{2, \mu}(\Omega, \delta)}\|v\|_{W_{2, v+\beta / 2}^{1}(\Omega, \delta)}
\end{gathered}
$$

hold. The proofs of estimates (43) and (44) are established by analogy with (19) and (20), which we obtain by using conditions (35), (38), and (40) and Lemma 1.

Let us show that the bilinear form is $\dot{W}_{2, \nu+\beta / 2}^{1}$-elliptical in $\Omega$. We have

$$
\begin{aligned}
& b(u, u) \\
& =\sum_{k=1}^{2} \int_{\Omega}\left[a_{k k} \rho^{2 v}\left(\frac{\partial u}{\partial x_{k}}\right)^{2}+a_{k k} \frac{\partial \rho^{2 v}}{\partial x_{k}} \frac{\partial u}{\partial x_{k}} u\right] d x \\
& \quad+\int_{\Omega} a \rho^{2 v} u^{2} d x
\end{aligned}
$$

for any $u$ from $\stackrel{\circ}{W}_{2, v+\beta / 2}^{1}(\Omega, \delta)$. By means of condition (35), we estimate the absolute value of the second term on the righthand side in (45):

$$
\begin{aligned}
\left|\sum_{k=1}^{2} \int_{\Omega} a_{k k} \frac{\partial \rho^{2 v}}{\partial x_{k}} \frac{\partial u}{\partial x_{k}} u d x\right| & \leq\left|\sum_{k=1}^{2} \int_{\Omega^{\prime}} a_{k k^{2}} 2 v \rho^{2 v-1} \frac{\partial u}{\partial x_{k}} u d x\right| \\
& \leq \varepsilon \sum_{k=1}^{2} \int_{\Omega^{\prime}} \rho^{2 v+\beta}\left(\frac{\partial u}{\partial x_{k}}\right)^{2} d x \\
& +\frac{2 c_{16}^{2} \nu^{2}}{\varepsilon} \int_{\Omega^{\prime}} \rho^{2 v+\beta-1} u^{2} d x
\end{aligned}
$$


From (45) and the last inequality we get

$$
\begin{aligned}
b(u, u) \geq & \sum_{k=1}^{2} \int_{\Omega} a_{k k} \rho^{2 v}\left(\frac{\partial u}{\partial x_{k}}\right)^{2} d x \\
& -\varepsilon \sum_{k=1}^{2} \int_{\Omega^{\prime}} \rho^{2 v+\beta}\left(\frac{\partial u}{\partial x_{k}}\right)^{2} d x \\
& +\int_{\Omega} a \rho^{2 v} u^{2} d x-\frac{2 c_{16}^{2} \nu^{2}}{\varepsilon} \int_{\Omega^{\prime}} \rho^{2 v+\beta-1} u^{2} d x .
\end{aligned}
$$

Supposing that $\alpha^{*}$ and $\alpha$ equal $\nu$ and $\nu / 2$ in Lemma 1 , respectively, we have

$$
\begin{array}{r}
\|u\|_{L_{2, \nu+\beta / 2-1}\left(\Omega^{\prime}, \delta\right)}^{2} \leq \frac{2 c_{6}^{2} \delta^{\nu}}{v}\|u\|_{L_{2, v+\beta / 2}(\Omega, \delta)}^{2}, \\
c_{6}=\text { const }>0 .
\end{array}
$$

Taking into account (36), (37), and (48), from estimate (47) we get

$$
\begin{aligned}
b(u, u) \geq & \left(c_{18}-\varepsilon\right)|u|_{W_{2, \nu+\beta / 2}^{1}(\Omega, \delta)}^{2}+\left(c_{19}-\frac{4 c_{16}^{2} c_{6}^{2} \nu \delta^{\nu}}{\varepsilon}\right) \\
& \times\|u\|_{L_{2, \nu+\beta / 2}(\Omega, \delta)}^{2} .
\end{aligned}
$$

Obviously, we can always choose $\varepsilon$ and $\delta$ such that the constants $c_{18}>\varepsilon, c_{19}>4 c_{16}^{2} c_{6}^{2} \nu \delta^{\nu} / \varepsilon$ and the inequality

$$
b(u, u) \geq c_{23}\|u\|_{W_{2, v+\beta / 2}^{1}(\Omega, \delta)}^{2}
$$

are valid with constant $c_{23}=\min \left(c_{18}-\varepsilon, c_{19}-4 c_{16}^{2} c_{6}^{2} \nu \delta^{\nu} / \varepsilon\right)$. Therefore, bilinear form $b(u, u)$ is $\stackrel{\circ}{W}_{2, v+\beta / 2}^{1}$-elliptical.

According to (43), (44), and (50), the bilinear form $b(u, u)$ is continuous and $\dot{W}_{2, \nu+\beta / 2}^{1}$-elliptical, and the linear form $l(v)$ is continuous on $\stackrel{\circ}{W}_{2, \nu+\beta / 2}^{1}(\Omega, \delta)$; then, the existence and uniqueness of an $R_{v}$-generalized solution of Problem B follow from the Lax-Milgram theorem (see [13]).

Taking into account that

$$
\begin{aligned}
c_{23}\left\|u_{\nu}\right\|_{W_{2, \nu+\beta / 2}^{1}(\Omega, \delta)}^{2} & \leq b\left(u_{\nu}, u_{\nu}\right) \\
& =l\left(u_{\nu}\right) \leq c_{22}\left\|u_{\nu}\right\|_{W_{2, v+\beta / 2}^{1}(\Omega, \delta)}\|f\|_{L_{2, \mu}(\Omega, \delta)},
\end{aligned}
$$

we get estimate (42).

Theorem 10. If for some $\delta$ there is a set of values $v$ such that an $R_{v}$-generalized solution of the Problem $B$ exists in the set $\stackrel{\circ}{W}_{2, v+\beta / 2}^{1}(\Omega, \delta)$, then this solution is unique for all such $v$.

The proof of this statement is similar to that of Theorem 2 in [14].

\section{Conflict of Interests}

The authors declare that there is no conflict of interests regarding the publication of this paper.

\section{References}

[1] F. Assous, P. Ciarlet Jr., and J. Segré, "Numerical solution to the time-dependent Maxwell equations in two-dimensional singular domains: the singular complement method," Journal of Computational Physics, vol. 161, no. 1, pp. 218-249, 2000.

[2] F. Assous, J. Ciarlet, E. Garcia, and J. Segré, “Time-dependent Maxwell's equations with charges in singular geometries," Computer Methods in Applied Mechanics and Engineering, vol. 196, no. 1-3, pp. 665-681, 2006.

[3] M. Costabel and M. Dauge, "Weighted regularization of Maxwell equations in polyhedral domains," Numerische Mathematik, vol. 93, no. 2, pp. 239-277, 2002.

[4] M. Costabel, M. Dauge, and C. Schwab, "Exponential convergence of hp-FEM for Maxwell equations with weighted regularization in polygonal domains," Mathematical Models \& Methods in Applied Sciences, vol. 15, no. 4, pp. 575-622, 2005.

[5] D. Arroyo, A. Bespalov, and N. Heuer, "On the finite element method for elliptic problems with degenerate and singular coefficients," Mathematics of Computation, vol. 76, no. 258, pp. 509-537, 2007.

[6] H. Li and V. Nistor, "Analysis of a modified Schrödinger operator in 2D: regularity, index, and FEM," Journal of Computational and Applied Mathematics, vol. 224, no. 1, pp. 320-338, 2009.

[7] V. A. Rukavishnikov, "On differentiability properties of an $R_{v}$ generalized solution of the Dirichlet problem," Soviet Mathematics. Doklady, vol. 309, no. 6, pp. 653-655, 1990, translated from Doklady Akademii Nauk SSSR, vol. 309, no. 6, pp. 13181320, 1989.

[8] V. A. Rukavishnikov, "On the Dirichlet problem for a secondorder elliptic equation with noncoordinated degeneration of the initial data," Differential Equations, vol. 32, no. 3, pp. 406-412, 1996.

[9] V. A. Rukavishnikov and E. V. Kuznetsova, "A coercive estimate for a boundary value problem with noncoordinated degeneration of the input data," Differential Equations, vol. 43, no. 4, pp. 550-560, 2007.

[10] V. A. Rukavishnikov, "Methods of numerical analysis for boundary value problems with strong singularity," Russian Journal of Numerical Analysis and Mathematical Modelling, vol. 24, no. 6, pp. 565-590, 2009.

[11] V. A. Rukavishnikov and H. I. Rukavishnikova, "The finite element method for a boundary value problem with strong singularity," Journal of Computational and Applied Mathematics, vol. 234, no. 9, pp. 2870-2882, 2010.

[12] V. A. Rukavishnikov and H. I. Rukavishnikova, "On the error estimation of the finite element method for the boundary value problems with singularity in the Lebesgue weighted space," Numerical Functional Analysis and Optimization. An International Journal, vol. 34, no. 12, pp. 1328-1347, 2013.

[13] P. G. Ciarlet, The Finite Element Method for Elliptic Problems, North-Holland, Amsterdam, The Netherlands, 1978.

[14] V. A. Rukavishnikov and A. G. Ereklintsev, "On the coercivity of the $R_{v}$-generalized solution of the first boundary value problem with coordinated degeneration of the input data," Differential Equations, vol. 41, no. 12, pp. 1757-1767, 2005. 


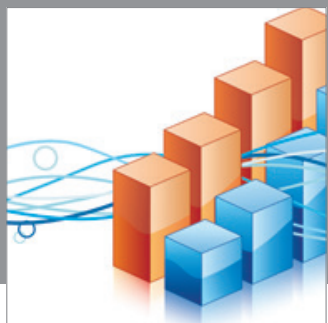

Advances in

Operations Research

mansans

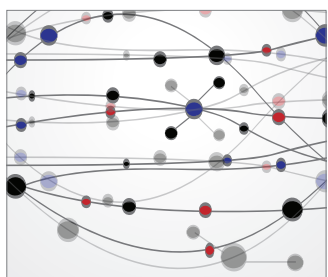

The Scientific World Journal
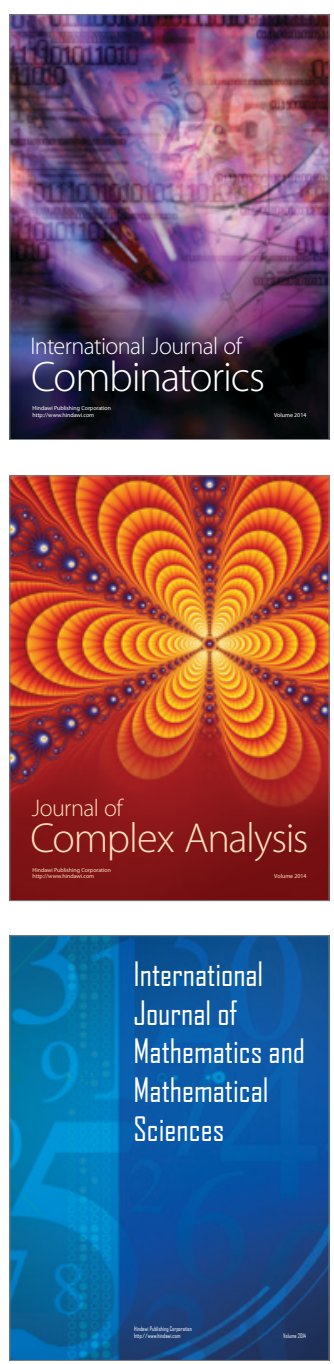
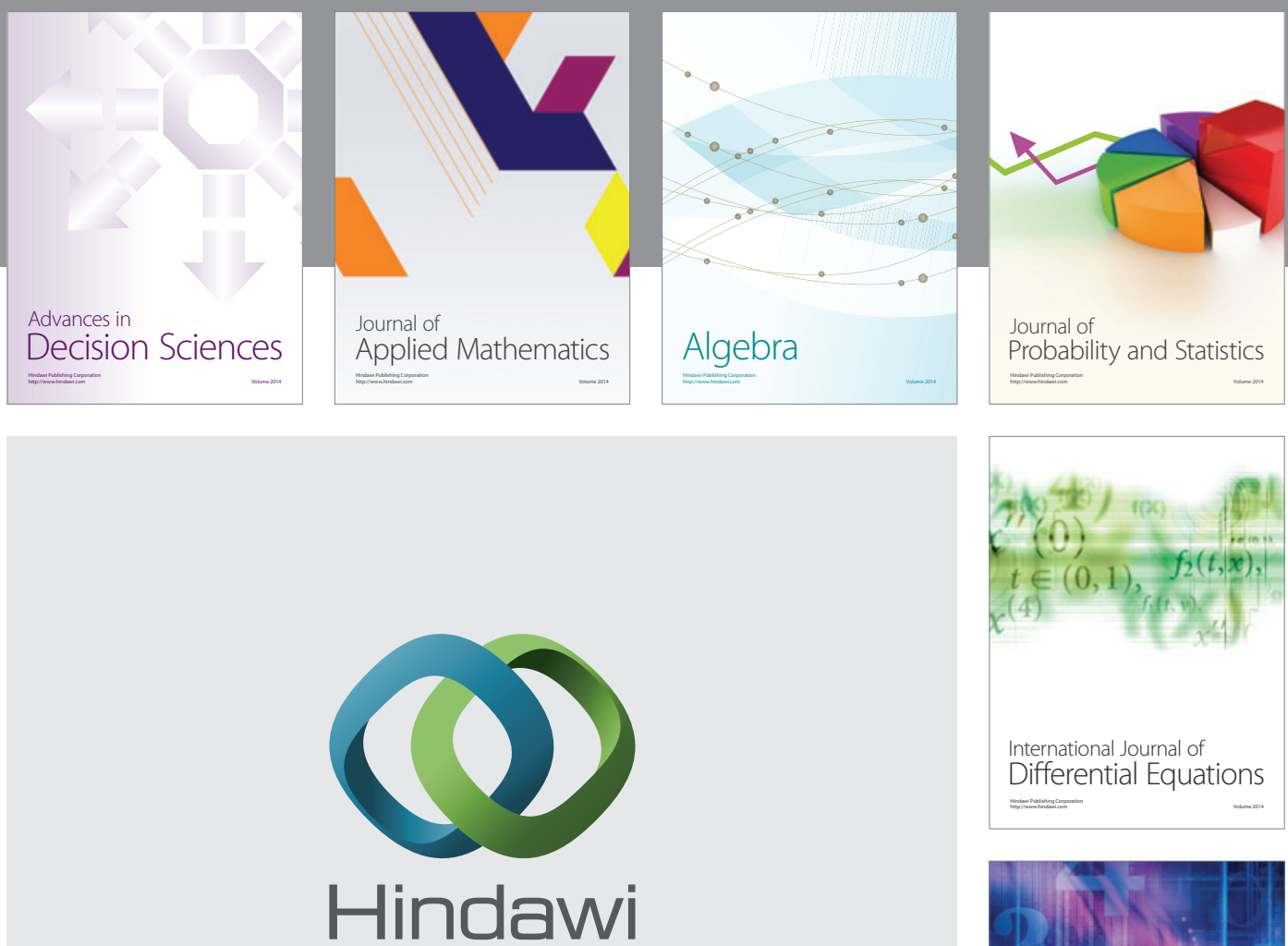

Submit your manuscripts at http://www.hindawi.com
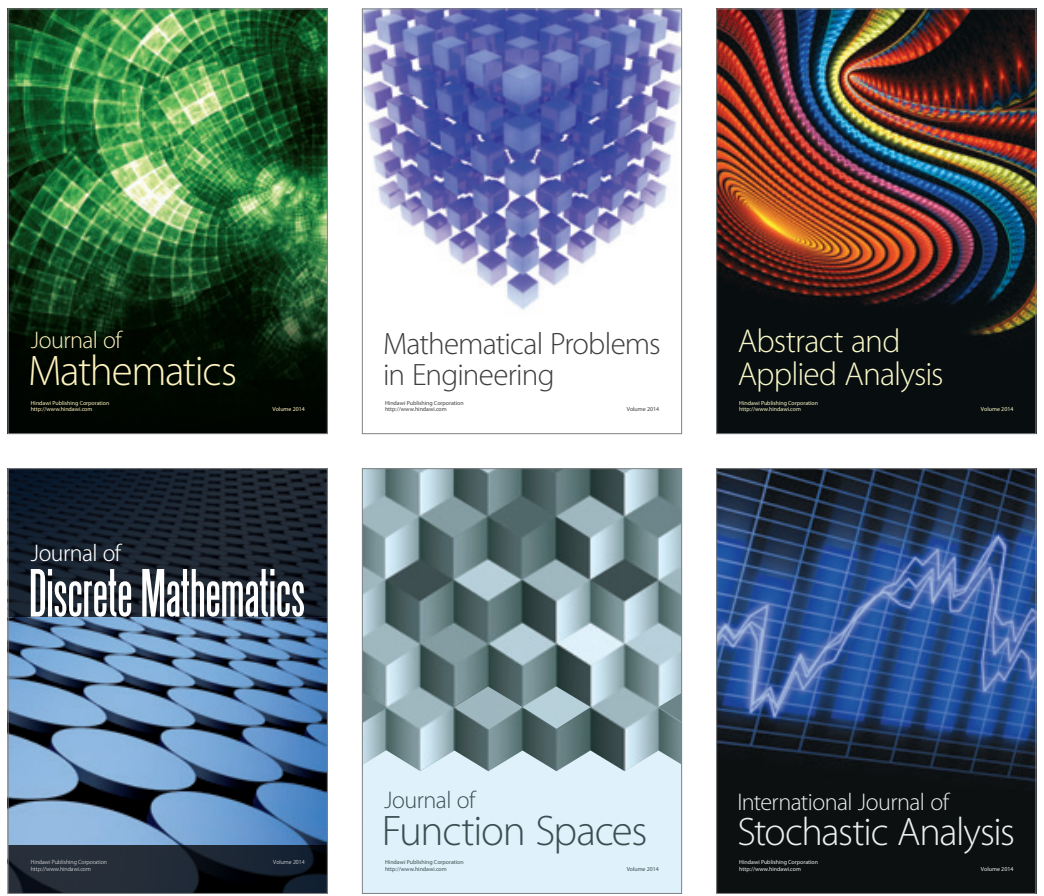

Journal of

Function Spaces

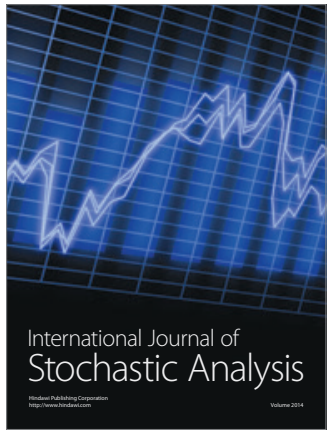

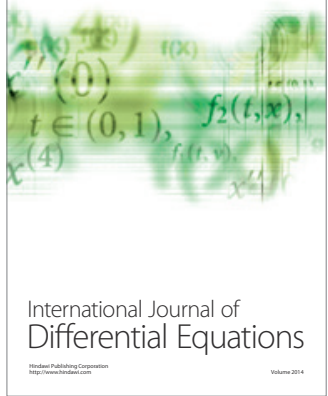
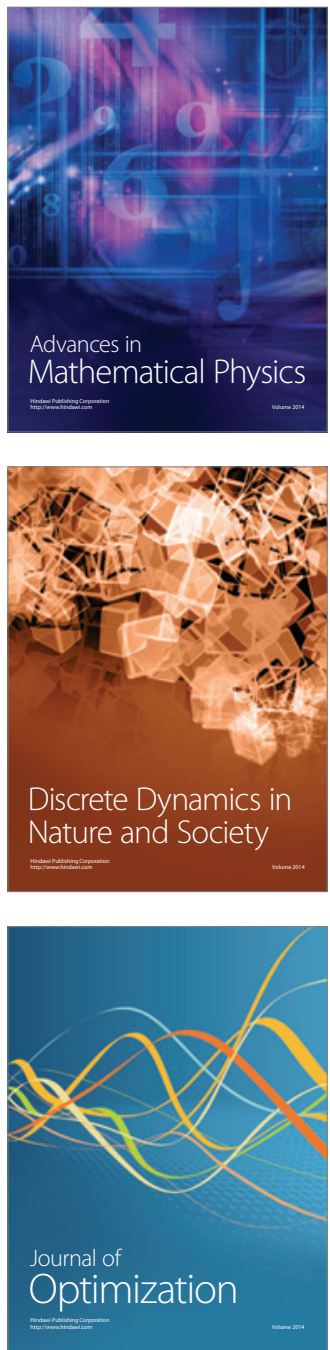